\title{
Capillary surfaces in an exotic container: results from space experiments
}

\author{
By P A UL CONCUS, ${ }^{1}$ ROBERT FIN N, ${ }^{2}$ \\ AND MARK WEISLOGEL ${ }^{3} \dagger$ \\ ${ }^{1}$ Lawrence Berkeley National Laboratory and Department of Mathematics, University of \\ California, Berkeley, CA 94720, USA \\ ${ }^{2}$ Department of Mathematics, Stanford University, Stanford, CA 94305-2125, USA \\ ${ }^{3}$ NASA Lewis Research Center, Cleveland, OH 44135, USA
}

Experimental results from the Interface Configuration Experiment (ICE) performed aboard the Space Shuttle and the Mir Space Station are reported. The experiment concerns fluid interfaces in certain "exotic" containers in a low-gravity environment. These containers are rotationally symmetric and have the property that for given contact angle and liquid volume, a continuum of distinct rotationally symmetric equilibrium configurations can appear, all of which have the same mechanical energy. These symmetric equilibrium configurations are unstable, in that deformations that are not rotationally symmetric can be shown mathematically to yield configurations with lower energy. It is found experimentally, in confirmation of mathematical results and of numerical computations, that distinct locally stable configurations can form that are not rotationally symmetric and have differing dynamic characteristics. It is found that this intriguing phenomenon of asymmetric local energy minimizers can occur even if conditions for an exotic container are not entirely met.

\section{Introduction}

It is essential, when planning space-based operations, to be able to predict the configurations that fluids will assume in their containers under low-gravity conditions. In such an environment surface wettability and container geometry predominate in governing equilibrium behaviour. As formulated in the classical Young-Laplace-Gauss (YLG) theory of capillarity, the wetting characteristics of a particular system are embodied in a well-defined equilibrium macroscopic contact angle depending only on the materials (see, e.g., Finn 1986, Chap. 1). The YLG theory characterizes equilibrium fluid configurations as those providing stationary values of the surface-plus-gravitational mechanical energy; a configuration is stable if it provides, as well, a local minimum of the energy.

Even under the simplifications of zero gravity (or low-gravity conditions for which the gravitational energy can be neglected), the strongly nonlinear governing equations from the classical theory are difficult to solve. Closed-form solutions for this case have been obtained for only a few simple configurations, such as in right circular cylindrical or spherical containers. Departures from these container geometries can lead to dramatic changes in fluid behavior (Concus \& Finn 1990).

Particular containers of interest are the "exotic" containers, which are discussed in

$\dagger$ Present address: TDA Research, Inc., 12345 W. 52nd Ave., Wheat Ridge, CO 80033, USA. 
(Concus \& Finn 1991) from a theoretical point of view (based on the YLG theory). These containers are rotationally symmetric with the remarkable property that for given contact angle and liquid volume, an infinity (in fact, an entire continuum) of distinct rotationally symmetric equilibrium configurations can appear, all of which have the same mechanical energy. Exotic containers are possible for any gravity level, but only for lowgravity conditions can the phenomena be expected to appear on an adequately large size scale to be readily measurable. For zero gravity, the case of principal interest here, the size scale can be arbitrary. Additionally, these containers can possess marked symmetrybreaking properties, as discussed below.

We describe in this paper the results of space experiments that investigate the actual physical behavior of liquids in exotic containers. Of central interest is whether the striking mathematical results based on the the fully nonlinear YLG classical theory can be observed experimentally in the presence of practical influences not reflected in that theory, such as contact angle hysteresis and dynamic contact line phenomena. Concurrently we seek indications as to the effects these factors may have on fluid behavior.

\section{Background}

\subsection{Theory}

According to the YLG formulation, equilibrium configurations of an incompressible liquid partly filling a container are stationary values, subject to the constraint of fixed liquid volume, of the energy expression (sum of capillary and gravitational potential energies)

$$
E=\sigma\left(\mathcal{S}-\mathcal{S}^{*} \cos \gamma\right)+\text { gravitational energy. }
$$

Here $\sigma$ is the liquid-gas interfacial tension and $\cos \gamma$ is the liquid-solid-gas relative adhesion coefficient, $\gamma$ being the contact angle between the liquid and the container; $\mathcal{S}$ denotes the area of the liquid-gas free surface and $\mathcal{S}^{*}$ the area of the portion of the container in contact with the liquid. The quantities $\sigma$ and $\gamma$ depend only on the materials, and are independent of the container geometry and gravitational field. Equilibrium configurations are stable, as well, if $E$ is locally minimized.

In (Concus \& Finn 1991) exotic rotationally symmetric container shapes are calculated numerically from the above equation and from the requirement that there be a continuum of rotationally symmetric liquid-gas free surfaces all having the same contact angle and enclosing the same volume of liquid with the container. Such container shapes, first studied by Gulliver \& Hildebrandt (1986) for the special case of zero gravity and contact angle $\pi / 2$, can be obtained for any contact angle or gravity level. A horizontal planar free surface making the specified contact angle with the container is a member of each family.

Figure 1 depicts zero-gravity exotic container meridians for a range of values of contact angle $\gamma$. The curves are scaled and shifted, so that in figure 1(a) they have a common lower left extremity at $r=1, z=0$ and so that in figure $1(b)$ they have a common vertical, rightmost extremity. The change in shape of the curves with $\gamma$ can be seen as particularly small near the common point in figure 1(a) and generally to decrease significantly as $\gamma \rightarrow 90^{\circ}$.

The closed container depicted in figure 2 is obtained by joining right circular cylindrical extensions to a calculated exotic container shape, in this case for zero gravity and contact angle $80^{\circ}$. The dashed curves depict selected members of the continuum of rotationally symmetric equilibrium interfaces, each enclosing volume $V$ with the container and having contact angle $\gamma$. The fluid configuration with planar free surface $\mathcal{P}$ is shown shaded. It is 

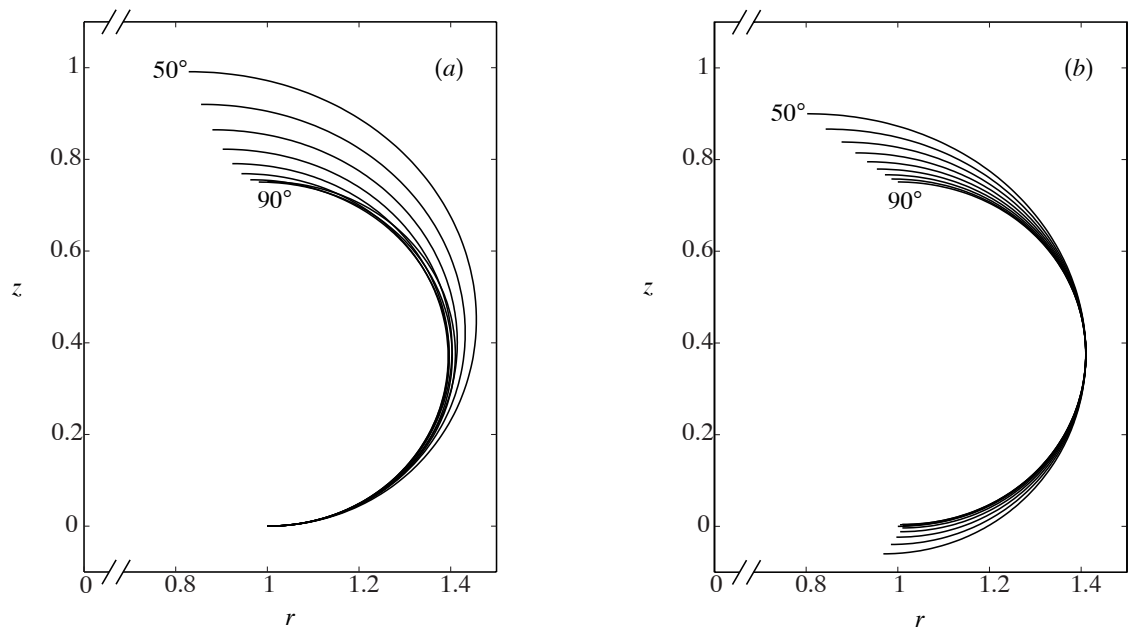

FiguRE 1. Overlay of zero-gravity exotic container meridians for contact angles $50^{\circ}$ to $90^{\circ}$, in $5^{\circ}$ increments. Axis of rotation is $r=0$. Meridians are scaled and shifted: $(a)$ to have a common lower left (horizontal) extremity at $r=1, z=0 ;(b)$ to have a common vertical rightmost extremity.

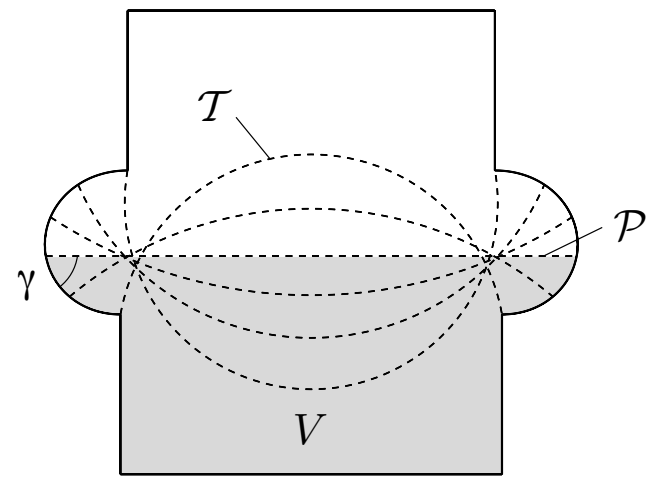

Figure 2. Axial section of exotic container for zero gravity and $\gamma=80^{\circ}$. Dashed curves depict members of continuum of rotationally symmetric equilibrium surfaces all of which enclose the same volume $V$ with the container. Topmost $(\mathcal{T})$ and planar $(\mathcal{P})$ symmetric members are indicated. The volume for the planar interface configuration is shown shaded.

an equilibrium configuration not only for zero gravity but also for any non-zero vertically directed gravity level. The topmost curved interface, discussed in ensuing sections, is denoted by $\mathcal{T}$.

As was shown by Finn (1988) and Concus \& Finn (1989), the rotationally symmetric equilibrium configurations turn out to be unstable; particular deformations that are not rotationally symmetric yield configurations with lower energy. Thus, it is possible to demonstrate a symmetric container that admits infinitely many symmetric equilibrium interfaces, but for which no interface that minimizes energy can be symmetric. This is in notable contrast with what happens in the familiar case of the right circular cylinder, for which the symmetric interface (unique for prescribed liquid volume) is stable, and no asymmetric ones can appear.

There is at present no known way to determine mathematically the surfaces that min- 
a)

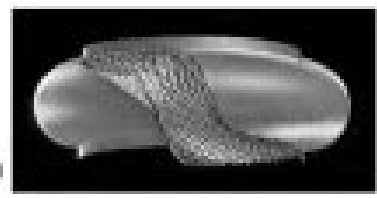

b)

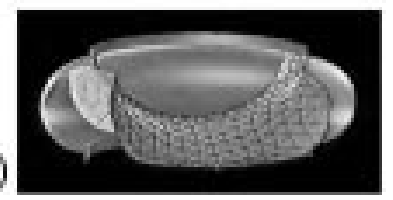

c)

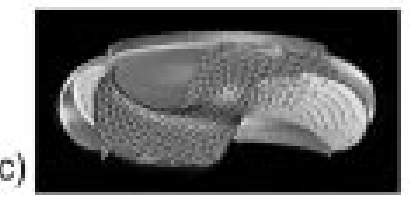

d)

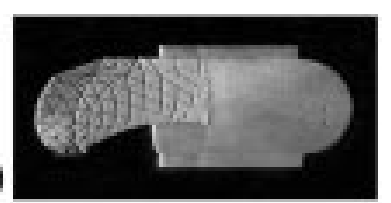

FiguRE 3. Computed stable surfaces in an exotic container for zero gravity and $\gamma=80^{\circ}$, in order of increasing energy: (a) "spoon", (b) "potato chip", $(c)$ "lichen". The rotationally-symmetric equilibrium family have larger energy. Orthogonal view of potato chip is shown in $(d)$.

imize energy in the exotic containers. However, numerical computations have suggested a number of particular surfaces not having rotational symmetry as local minima. Using a modification of an early version of the Surface Evolver software package (Brakke, 1996), Callahan, Concus \& Finn (1991) computed three distinct non-rotationally-symmetric local energy minimizing surfaces for exotic containers such as the one shown in figure 2 . The surfaces, all of which yield smaller energy than that of the symmetric equilibrium family, are depicted in figure $3(a-c)$ in order of increasing energy. To expose the surfaces to view, only half of the container, lying to one side of a vertical plane through its axis of symmetry, is shown in figure 3. The container's exotic-bulge portion and just a small length of the circular cylindrical extensions are depicted. The liquid would lie below the free surface interfaces.

We refer to the surface configurations as the "spoon" (figure $3 a$ ), the "potato chip" (figure $3 b$ ), and the "lichen" (figure $3 c$ ). The contact lines of these surfaces with the container undergo respectively one, two, and three excursions from upper to lower extremities and back as they circumnavigate the bulge. Figure $3(d)$ depicts an orthogonal view of the potato chip configuration of figure $3(b)$. The surfaces were obtained in each case by starting the computational iterations from an initial approximation of the horizontal planar member of the symmetric equilibrium interface continuum to which a small volume-preserving perturbation had been added. The three local energy minimizing surfaces were found by varying the form of the initial perturbation. The computed (non-dimensional) energies are 0.47, 0.69, and 0.86, respectively, for a container scaled so that the symmetric family (figure 2) have energy unity. For ease of comparison, these energy values exclude the contributions from the lower cylindrical wall portions used in the computations and from the base (wall and base are entirely covered with liquid in the configurations). The mean curvatures $H$ of the individual stationary configurations in general differ from one another. Correspondingly, the associated capillary pressures (i.e., pressure changes $\Delta p=2 \sigma H$ across the free surfaces) will differ. This is the case for the above asymmetric minimizing configurations and also, as is readily apparent from figure 2 , for the symmetric equilibrium family.

\subsection{Drop tower experiment}

As it is not clear to what extent the idealizations of the formal theory are reflected in reality, it is important to determine experimentally what physically observable locally energy-minimizing configurations there might be. In this regard, an experimental study 

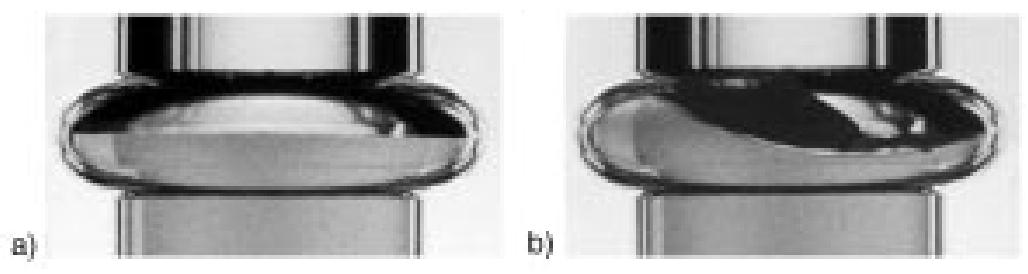

FiguRE 4. Drop tower results: (a) Initial (1-g) planar interface; (b) Configuration after $5.1 \mathrm{~s}$ of free fall $\left(\approx 10^{-5} \mathrm{~g}\right)$. (Spoon) configuration in $(b)$ has not completely stabilized.

was initiated with the primary objective of investigating energy-minimizing configurations in the exotic containers. As mentioned in $\S 1$, even though exotic containers are possible for any gravity level, container dimensions become of adequately large scale for practical construction only for gravity approaching zero. Thus, low-gravity experiments were proposed. They were conducted initially in the NASA Lewis Research Center 5.1second drop tower for the $80^{\circ}$ zero-gravity exotic container depicted in figure 2 . The liquid in the container was distilled water, for which the equilibrium contact angle with the acrylic material of the container was measured by the wedge method (Concus \& Finn 1974, pp. 220-221) to be $80^{\circ}$, repeatable to within $\pm 2^{\circ}$.

The results of these tests were reported by Concus, Finn \& Weislogel (1992). Although insufficient low-gravity time was available for a fully static interface to be achieved, it was found that the initial 1-g interface of the planar configuration $\mathcal{P}$ in the continuum family reoriented during the approximately five seconds of weightlessness toward an asymmetric configuration much like the spoon of figure 3(a). These initial and low- $g$ surfaces are shown in figure 4 . In several other of the tests no reorientation of the fluid occurred, and the surface remained essentially planar. Whether the fluid did or did not reorient appears to have depended on the nature of spurious disturbances imparted to the container during the transition between 1- $g$ and low- $g$.

Drop tower tests to study the effects of imprecise fluid volume and initial tilt angle with respect to gravity were performed also. These indicated that reorientation to the spoon-like surface shapes could still occur, even though conditions for an exotic container were not entirely met. This is in accord with computational evidence that the asymmetric configuration phenomenon generally is robust with respect to deviation of parameters (contact angle, liquid volume, gravity level) from their precise exotic values, to discretization errors from numerical approximations, and to the type of inaccuracies inherent in container fabrication.

Because the drop tower tests could offer observation of the actual behavior of the fluid for only a brief interval of time, information concerning the local stability of any static low- $g$ interface configuration, such as the spoon, could not be obtained.

\section{Space experiments}

Based on the mathematical and computational results and on the observations made in the drop-tower experiment, the in-space Interface Configuration Experiment (ICE) was proposed. The extended low-gravity environment of an orbiting spacecraft would provide an opportunity for attempts to observe one or more of the asymmetric locally stable equilibria suggested by the numerical computations and to examine their stability and dynamic characteristics. The possibility that in a container that is rotationally symmetric there can be multiple locally-stable nonaxisymmetric capillary surfaces, each of which 


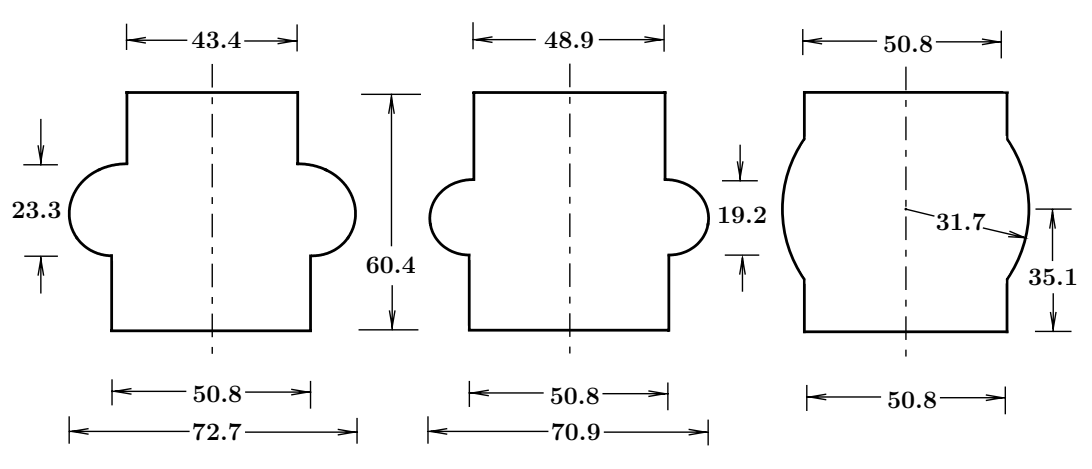

(a)

(b)

$(c)$

Figure 5. Axial sections of containers for experiment: (a) vessels 1 and 2, (b) vessel 3, (c) vessel 4 . Indicated dimensions are in $\mathrm{mm}$.

may possess distinct dynamic characteristics, has particular interest for designers of inspace fluids systems.

ICE was carried out in two segments, one aboard the NASA Space Shuttle during the First United States Microgravity Laboratory flight (USML-1/STS-50) and the other on the Russian Space Station Mir during the Mir-21/NASA-2 mission. The experiment was performed in the glovebox, a multi-user facility developed by the European Space Agency for experiments on Spacelab missions, on the Space Shuttle middeck, and on Space Station Mir. $\dagger$ The experiment was ideally suited for the glovebox because of the simplicity of the apparatus and the opportunity for hands-on interaction by the crew.

The exotic containers could readily be incorporated into a test apparatus suitable for in-space experiments. Four vessels were constructed, of which all were flown aboard USML-1 and two re-flown aboard Mir. The exotic containers were designed for the case of zero gravity, as in the drop tower experiment, and for two different liquids. Figure $5(a)$ shows an exotic container for the case of a $55^{\circ}$ contact angle (vessels 1 and 2); figure $5(b)$ shows a container for an $80^{\circ}$ contact angle (vessel 3), the same one as for the drop tower experiment; the container in figure $5(c)$ has a spherical bulge and was used as a control (vessel 4).

The four vessels are similar in construction; a vessel flight unit is shown in figure 6 . The primary vessel components are a single-piece (transparent) acrylic-plastic body (outer dimensions $90 \times 90 \times 184 \mathrm{~mm}$ and mass $1.5 \mathrm{~kg}$ ), a fluid reservoir with aluminium piston and volume control dial, and a two-port aluminum valve. O-ring seals are used throughout. A diamond-tipped cutting tool mounted to a numerically controlled airbearing lathe was used to machine the vessels.

The exotic container coordinates (in $\mathrm{mm}$ ) fed to the lathe are given in Table 1 for vessels 1,2 , and 3; these correspond to curves depicted in figure 1(a). The coordinates (specified for each ten degrees change in slope) were interpolated by the lathe with cubic splines. After being machined, the surfaces were finished with a light pass of a dry cloth (without polish). The resulting profiles after annealing deviated less than $\pm 50 \mu \mathrm{m}$ from

$\dagger$ Originally designed to handle biological experiments, the glovebox has been adapted to handle fluids, combustion, and materials science experiments and has served well in providing a rapidly accessible, inexpensive platform in which to conduct experiments in space. ICE utilized the glovebox primarily as a staging area and as a level of containment in the event of a fluid spill. 


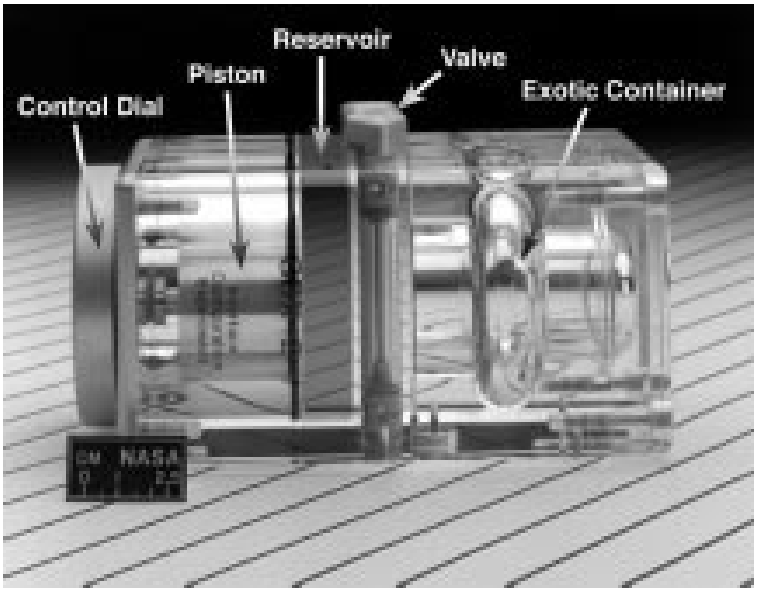

Figure 6. ICE vessel, flight unit.

\begin{tabular}{cccccccccc} 
& \multicolumn{4}{c}{ Vessels $1 \& 2$} & \multicolumn{2}{c}{ Vessel 3} & \multicolumn{4}{c}{ Vessels $1 \& 2$} & \multicolumn{2}{c}{ Vessel 3} \\
pt. & $r$ & $z$ & $r$ & $z$ & pt. & $r$ & $z$ & $r$ & $z$ \\
1 & 25.400 & 19.050 & 25.400 & 19.050 & 11 & 36.192 & 31.693 & 35.338 & 29.970 \\
2 & 27.440 & 19.227 & 27.386 & 19.222 & 12 & 35.672 & 33.628 & 34.923 & 31.517 \\
3 & 29.336 & 19.734 & 29.190 & 19.703 & 13 & 34.791 & 35.515 & 34.229 & 33.002 \\
4 & 31.060 & 20.537 & 30.795 & 20.451 & 14 & 33.540 & 37.299 & 33.259 & 34.386 \\
5 & 32.586 & 21.605 & 32.186 & 21.423 & 15 & 31.914 & 38.923 & 32.016 & 35.628 \\
6 & 33.888 & 22.906 & 33.347 & 22.583 & 16 & 29.913 & 40.321 & 30.504 & 36.684 \\
7 & 34.939 & 24.408 & 34.267 & 23.896 & 17 & 27.541 & 41.424 & 28.733 & 37.509 \\
8 & 35.716 & 26.076 & 34.934 & 25.326 & 18 & 24.808 & 42.153 & 26.712 & 38.048 \\
9 & 36.198 & 27.872 & 35.339 & 26.838 & 19 & 21.729 & 42.418 & 24.453 & 38.242 \\
10 & 36.362 & 29.759 & 35.475 & 28.398 & & & & &
\end{tabular}

TABLE 1. Coordinates ( $\mathrm{mm}$ ) given to the lathe for construction of exotic containers for vessels 1 and 2 (identical) and vessel 3 . The base of the lower cylindrical extension of each container is centered at $r=z=0$.

the specified values at the coordinate points. In terms of a change in specified contact angle, this deviation would be comparable to approximately $\pm 0.02^{\circ}$ for the $55^{\circ}$ exotic container profile. The cubic spline interpolation errors were an order of magnitude less. Characteristic (interior) container dimensions are shown in figure 5. The joins between the bulge portions of the containers and the cylindrical extensions were machine rounded to a radius of $0.76 \mathrm{~mm}$.

The liquid employed in the experiments for the $55^{\circ}$ exotic containers (vessels 1 and 2 ) is an immersion oil, a blend of hydrogenated terphenyl and an alyphatic hydrocarbon supplied by Cargille Laboratories (code 5040). It possesses the special property of having a refractive index matched with that of the acrylic container $\left(N_{D}=1.491\right.$ at $\left.20^{\circ} \mathrm{C}\right)$, thereby keeping optical distortions minimal. A volume of $71.0 \mathrm{ml}$ of this fluid was placed in each of the vessels. To achieve the desired wetting conditions a fluorochemical polymer surface coat approximately $0.25 \mu \mathrm{m}$ thick (FC-723 manufactured by $3 \mathrm{M}$ Corporation) was applied to the interior walls of the exotic container portions of the vessels.

It was determined that a particular batch of immersion fluid, the one used on USML-1, had actually a blend yielding an equilibrium contact angle of $70^{\circ}$ with the coated surface 


\begin{tabular}{|c|c|c|c|c|c|c|c|}
\hline Liquid & Vessels & $\begin{array}{c}\rho \\
\mathrm{kg} \mathrm{m}{ }^{-3}\end{array}$ & $\begin{array}{c}\sigma \\
\mathrm{N} \mathrm{m}^{-1}\end{array}$ & $\mathrm{~m}^{2} \mathrm{~s}^{\nu 1}$ & $\begin{array}{c}\gamma \\
\pm 2^{\circ}\end{array}$ & $\begin{array}{l}\gamma_{a} \\
\pm 2^{\circ}\end{array}$ & $\begin{array}{l}\gamma_{r} \\
\pm 2^{\circ}\end{array}$ \\
\hline Immersion oil & 1,2 & 894 & 0.0324 & $31.0 \times 10^{-6}$ & 70 & 75 & 60 \\
\hline Distilled water & 3,4 & 1000 & 0.0713 & $1.08 \times 10^{-6}$ & 80 & 90 & 67 \\
\hline
\end{tabular}

TABLE 2. Test liquid data at room temperature; $\rho, \sigma$, and $\nu$ are density, surface tension, and kinematic viscosity, respectively. $\gamma$ is the measured equilibrium contact angle, and $\gamma_{a}$ and $\gamma_{r}$ the measured static advancing and receding values (for the respective vessel surfaces: immersion oil on FC-723 coated acrylic surface, distilled water on machined acrylic).

(with a hysteresis range of $60^{\circ}-75^{\circ}$ ) rather than the $55^{\circ}$ of earlier batches. As can be the case for space experiments, with their rigid time schedules and periods of limited opportunity for direct control, unplanned events may arise that require flexibility on the part of the investigators. In this case the contact angle discrepancy was viewed as an opportunity for testing exotic containers with a liquid whose contact angle departed measurably from that for which the containers were designed. This matter is discussed further in $\S 6$. The $70^{\circ}$ contact angle immersion fluid was used also for vessels 1 and 2 in the Mir experiments. The $71.0 \mathrm{ml}$ liquid volume given above is the one corresponding to the $70^{\circ}$ liquid, as determined in 1- $g$ by filling a container to the planar fill level (the shaded volume in figure 2).

Distilled water was used in vessels 3 and 4 . No coating was applied in these containers; the contact angle of the water on acrylic was measured to be $80^{\circ}$ with hysteresis in the range $67^{\circ}-90^{\circ}$, all angle measurements being accurate to $\pm 2^{\circ}$. The liquid volumes used, as determined by the 1- $g$ planar fill level procedure, were $68.1 \mathrm{ml}$ for vessel 3 and 78.5 $\mathrm{ml}$ for vessel 4 .

Both the immersion fluid and the water were lightly dyed to enhance visibility of the interfaces. Properties of the (dyed) fluids are listed in Table 2.

\section{Experiment procedures}

The general procedure for ICE was to partially fill the exotic container portion of a selected vessel and to record with two video cameras the fluid interface configurations that unfolded as the experiment progressed. A vessel being tested was first set up in the glovebox, the reservoir valve was then opened, and the control dial was turned to displace the entire contents of the reservoir into the exotic container portion of the vessel. After a sufficient time, disturbances were imparted to the vessel and the resulting interfaces were observed, generally until they become quiescent again. The disturbances were increased in amplitude until the interface either consistently returned to a particular configuration or broke up. The two video cameras recording the process were mounted for orthogonal views, and audio was recorded simultaneously. Glovebox temperature and local accelerometer data were obtained also. The specific procedures particular to the USML-1 and Mir experiments are treated separately below.

\subsection{Procedure for ICE USML-1}

As discussed in $\S 2.2$, the drop tower tests indicated that surfaces that form in exotic containers in low- $g$ may be sensitive to spurious disturbances. Thus, for the USML-1 experiments care was taken to mount the ICE vessels rigidly to the glovebox floor by means of a labjack. After the fluid was carefully dispensed into an exotic container in a manner that encouraged establishment of a configuration like that for the topmost 
member of the family of equipotential rotationally symmetric surfaces $(\mathcal{T}$ in figure 2$)$, the crew member then imparted a perturbing impulse to the liquid by tapping the outside of the vessel with his finger. The location of the tap was near the bulge of the exotic container (so that it could be captured on video) and was directed normal to the axis. The solitary taps began as light disturbances, allowing for complete stabilization of the interface between each tap. These lighter taps were intended to dislodge a surface that might otherwise be unstable, but was "sticking" in its configuration because of resistance at the contact line (hysteresis), much as the planar configuration stuck in some of the drop tower tests if spurious disturbances did not release it ( 2.2$)$. Subsequently, intensity of the taps was increased to test the stability of any configuration that might have formed.

\subsection{Procedure for ICE Mir}

The ICE vessels tested on Mir were rigidly mounted to a labjack, as were the USML-1 ones, however the labjack was itself not rigidly mounted to the glovebox floor. Instead, the labjack was held to the glovebox by means of magnets. Tapping, impulse-type disturbances were imparted to the vessels in a manner similar to that for the USML-1 experiment, but were directed along the axis of the vessel. To induce larger amplitude axial disturbances, the crew member could slide the vessel-labjack assembly back and forth along the glovebox floor.

\section{Results}

The results of ICE for USML-1 are summarized here briefly; for further particulars see (Concus, Finn \& Weislogel 1993). The results for the Mir experiments, which exhibit a wider range of behavior, are discussed here more fully.

\subsection{Results for ICE USML-1}

After the fill procedure for transferring the liquid from the reservoir to the container portion of the vessels was completed, the resulting configurations were like the one of the topmost symmetric interface $\mathcal{T}$ in figure 2. Such was the case for all four vessels. Subsequent light taps to the vessels produced high frequency waves on the liquid freesurface that dissipated rapidly. The amplitude of the surface oscillations was considerably less than $1 \mathrm{~mm}$ during this portion of the tests. For the exotic vessels 1, 2, and 3, from two to seven such taps of this nature eventually destabilized the initial rotationally symmetric surface, which then established a configuration like that of the spoon. The orientation of the spoon was not generally aligned with the orientation of the taps, which were imparted normal to the container axis. To increase the amplitude of subsequently imparted disturbances, the crew member found it necessary to rap on the side of the vessel with his knuckles, as the vessels were rigidly mounted. This procedure produced larger amplitude (up to $4 \mathrm{~mm}$ ) oscillations of the interface about the spoon configuration, but these oscillations decayed without noticeable reorientation of the liquid bulk. The USML1 results for the exotic containers thereby succeeded in demonstrating the existence of an asymmetric spoon-type configuration, in accordance with the computational and drop tower results, and in portraying its marked stability.

The container portion of the fourth vessel is not exotic but has a bulge that is a portion of a sphere, for which complete mathematical results are available. The infinite family of symmetric equilibrium interfaces that are possible for the exotic container bulges cannot form. However, by rigid-body rotation of the liquid, an equilibrium surface in zero- $g$ can be tilted as desired with respect to the axis of symmetry without any change in shape or energy. This tilting occurred during the tests once the interface was freed from its initial 


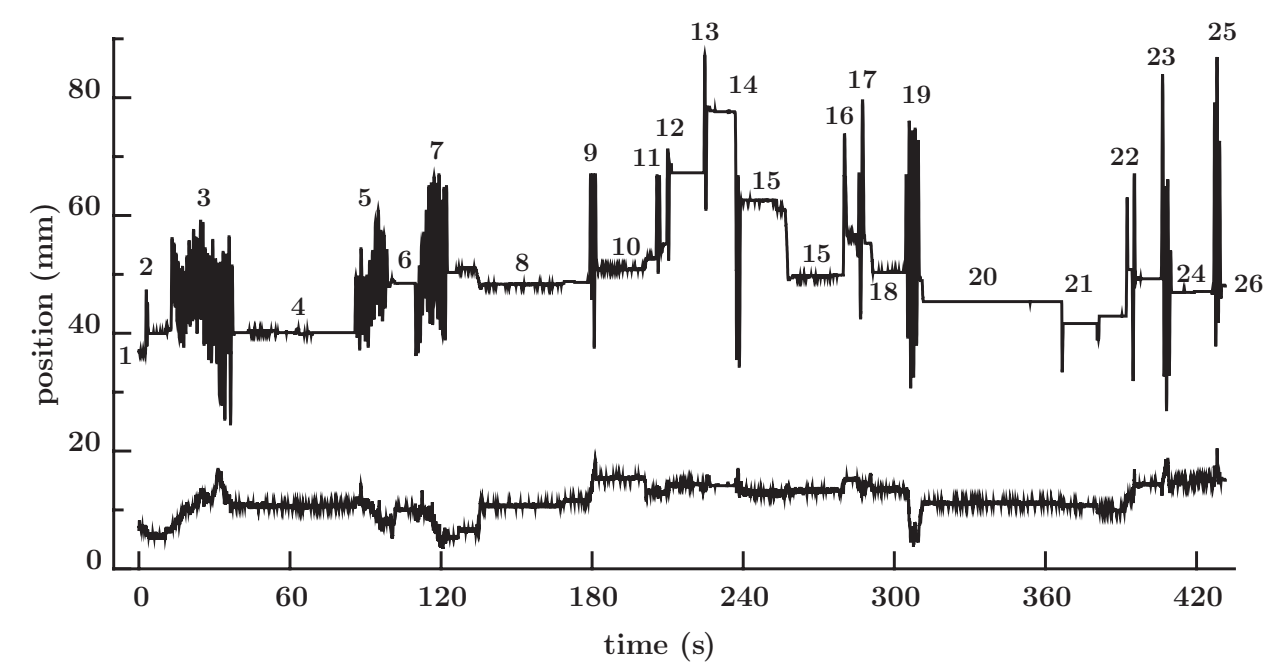

FiguRE 7. History of disturbances to vessel 2, ICE Mir; upper curve is for component directed along the container axis, lower curve is for lateral (orthogonal) component.

shape pinned at the join between the cylinder and the bulge. Subsequent disturbances merely led to further rotation or tilting of that configuration with no appreciable change to the interface shape (which was essentially planar, corresponding to the volume of liquid that was prescribed). Thus the interfaces for vessel 4 remained rotationally symmetric, though not necessarily about the vessel axis.

\subsection{Results for ICE Mir}

ICE vessels 1 and 2 were re-tested on Mir. The vessels were filled in the same manner as on USML-1, as specified in the general procedure in $\S 4$. Because the vessels were held with magnets to the glovebox floor in this case, rather than by rigid connection, the manual turning of the control dial during the fill produced measurable disturbances to the liquid. For example, for vessel 2, when the fill was near to being complete, the disturbances were found to be approximately $1.4 \mathrm{~mm}$ in amplitude over a time period of approximately $0.08 \mathrm{~s}$. The disturbances were not axially symmetric. At first, they excited waves across the surface of frequency $\omega \approx 1.87 \mathrm{~Hz}$, which then decayed to a fundamental, lower-frequency slosh mode of frequency $\omega_{n} \approx 0.90 \mathrm{~Hz}$ and characteristic amplitude $A \approx 0.8 \mathrm{~mm}$. Each disturbance required a time period $t_{s} \approx 8 \mathrm{~s}$ to settle to a quiescent state. These disturbances did not lead to instability of the interface.

Once the fill procedure was complete, the disturbances imparted by the crew member in removing her hands from the cell did result in destabilization of the symmetric surface and a reorientation to the spoon. The formation of the spoon was slow at first, requiring approximately $10 \mathrm{~s}$ after the initial perturbation (hands letting go) before it began to form. The bulk of the spoon formation was then completed in about another $3 \mathrm{~s}$; the remaining settling took about $3 \mathrm{~s}$ more. This was the case for both vessels 1 and 2 .

Once the spoon was formed, the crew member tapped lightly along the container axis. This light tapping again produced higher frequency waves that rapidly decayed to a lower frequency mode with $A<0.3 \mathrm{~mm}, \omega_{n} \approx 1.57 \mathrm{~Hz}$, and $t_{s} \approx 3 \mathrm{~s}$. The spoon configuration was not otherwise altered by the taps.

At this point the crew member imparted increasingly larger amplitude oscillations, 

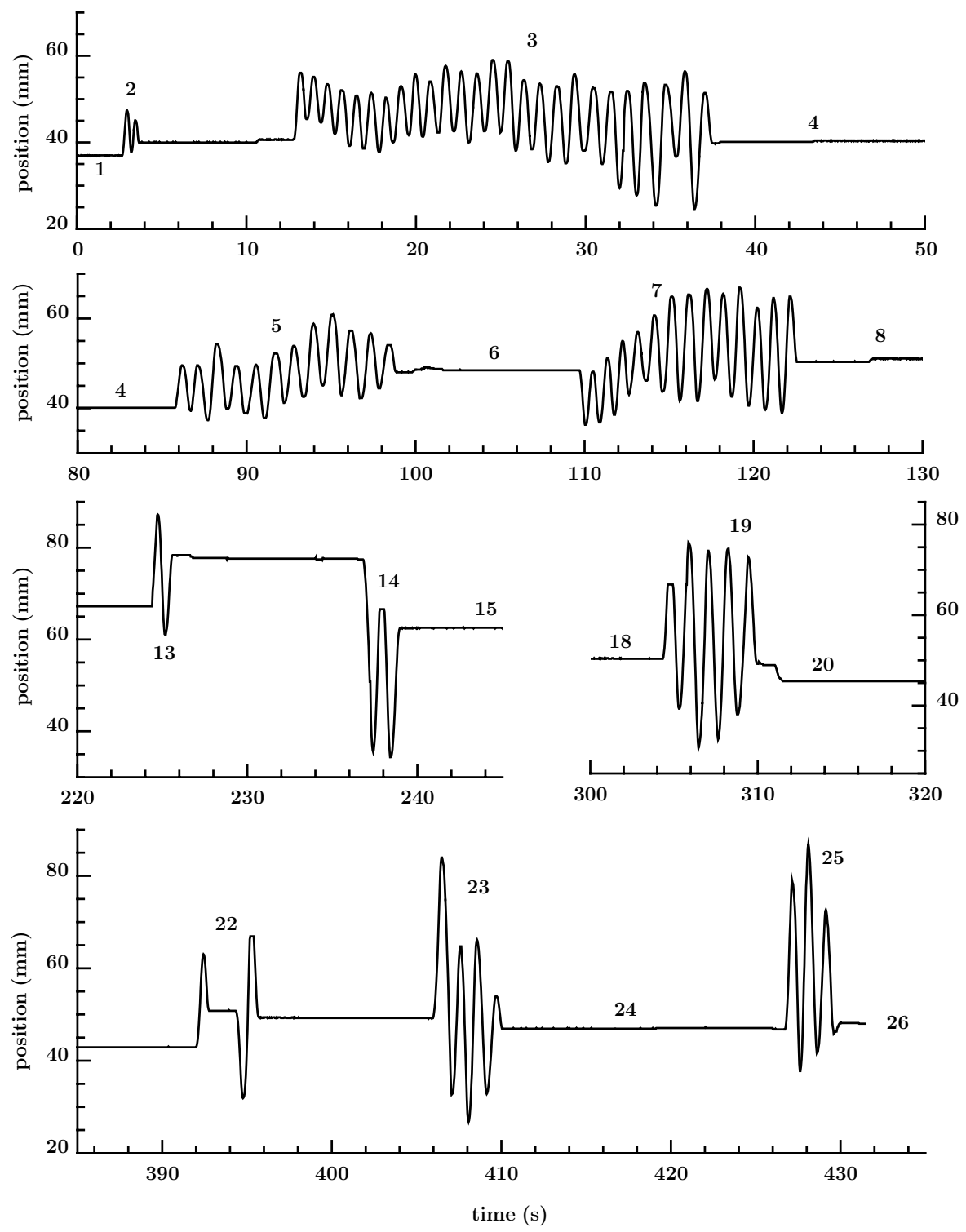

Figure 8. Selected time slices of vessel 2 history from figure 7.

periodic in nature, by sliding the vessel assembly back and forth along the glovebox floor. In figure 7, a history of these disturbances is presented for vessel 2 , the vessel in which a distinct, second locally stable configuration (potato chip) was subsequently induced to form. The data for figure 7 are obtained by tracking in time a fixed location on the vessel using an automated digital image analysis system (thirty video frames per second). The history begins after the first formation of the spoon. The relatively larger amplitudes of the upper curve in figure 7 (disturbances directed along the container axis) compared with those of the lower curve (lateral disturbances) illustrate the degree to which the large amplitude disturbances, imparted to the vessel by hand, were directed primarily along the container axis. Figure 8 shows expanded time scale slices from figure 7; figure 9 displays video images of selected interfaces, which are discussed below. 


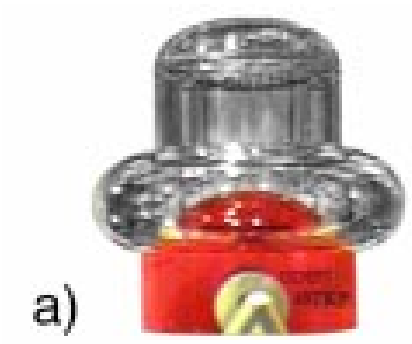

c)

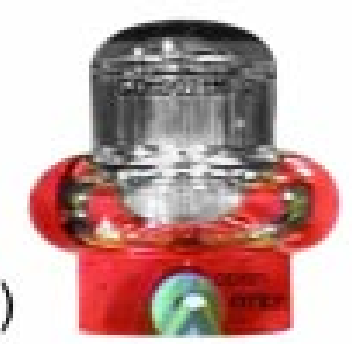

e)

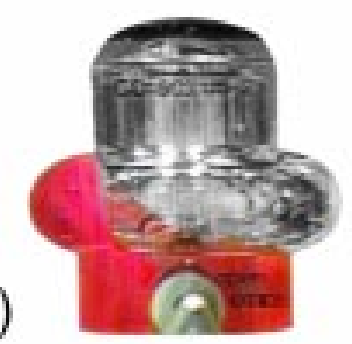

b)
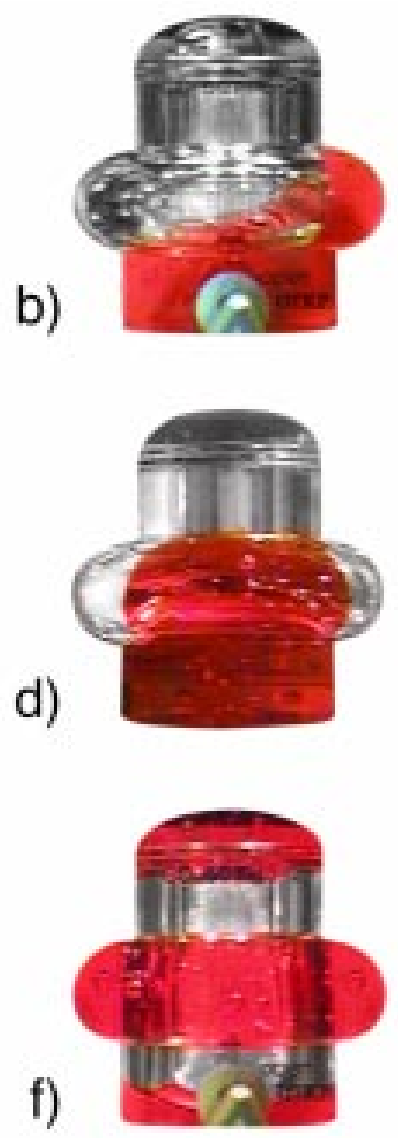

FiguRE 9. Static interface configurations during experiments: $(a)$ rotationally symmetric interface (pinned), (b) spoon-right, $(c)$ potato chip, $(d)$ orthogonal view of potato chip, $(e)$ spoon-left, and $(f)$ broken interface.

The initial spoon configuration at frame 0 on figure 7 is shown in figure $9(b)$ (cf. figure $3(a)$ ), to which we refer as the spoon-right configuration. This is the configuration to which the symmetric interface figure $9(a)$ reoriented after being destabilized. The events discussed in detail in the chronology below are the ones denoted by the numbers on figure 7 (and figure 8).

[1] Initial configuration. Prior to the large oscillations that begin at approximately frame 0 , a spoon configuration forms in the righthand portion of the bulge. Light taps along the vessel axis result in slosh-type surface oscillations of $\omega_{n} \approx 1.57 \mathrm{~Hz}, A<0.3$ $\mathrm{mm}$, and $t_{s} \approx 3 \mathrm{~s}$.

[2] First larger-oscillation-type disturbance to the spoon shape. Input disturbance of approximately two periods of oscillation at $1.54 \mathrm{~Hz}$ with an average amplitude of $A_{\text {ave }} \approx 11 \mathrm{~mm}$ gives rise to surface oscillations, with decay back to the spoon-right configuration.

[3-13] Sequence of disturbances to the spoon shape. During steps 3, 5, and 7, the large number of periods of oscillation $(25,11$, and 13 , respectively) act to make the dynamic interface more axially symmetric. For these three disturbances the average resonant frequencies are 1.04, 0.90, and 1.01, respectively. Maximum amplitude $A_{\max }$ and 


\begin{tabular}{|c|c|c|c|c|c|c|c|}
\hline Event & $\begin{array}{l}\omega_{n} \\
\mathrm{~Hz}\end{array}$ & $\begin{array}{l}\text { Periods of } \\
\text { Oscillation }\end{array}$ & $\begin{array}{c}A_{\max } \\
\mathrm{mm}\end{array}$ & $\begin{array}{l}A_{\text {ave }} \\
\mathrm{mm}\end{array}$ & $\begin{array}{l}\text { initial/subsequent } \\
\text { configurations }\end{array}$ & $\begin{array}{l}B o_{\omega} \\
\max \end{array}$ & $\begin{array}{r}B o_{\omega} \\
\text { ave }\end{array}$ \\
\hline$[1]$ & 0.90 & - & 0.8 & - & init. symmetric/spoon-right & 0.45 & - \\
\hline [3] & $1.06 \pm 0.15$ & 25.5 & 15.9 & $8.1 \pm 2.6$ & spoon-right/spoon-right & 12.6 & 6.4 \\
\hline [5] & $0.90 \pm 0.08$ & 11.5 & 8.9 & $6.4 \pm 1.2$ & spoon-right/spoon-right & 5.1 & 3.6 \\
\hline$[7]$ & $1.03 \pm 0.08$ & 13 & 13.1 & $9.5 \pm 2.7$ & spoon-right/spoon-right & 9.8 & 7.1 \\
\hline$[14]$ & $1.00 \pm 0.00$ & 1.5 & 21.0 & $16.6 \pm 3.0$ & spoon-right/unstable chip & 14.8 & 11.7 \\
\hline [19] & $0.86 \pm 0.05$ & 4 & 22.4 & $17.3 \pm 4.7$ & spoon-right/potato chip & 11.6 & 9.0 \\
\hline$[22]^{*}$ & 0.96 & $\lesssim 1$ & 17.8 & 17.8 & potato chip/potato chip & 11.5 & 11.5 \\
\hline$[23]$ & $0.94 \pm 0.11$ & 3 & 25.5 & $17.5 \pm 4.6$ & potato chip/spoon-left & 15.8 & 10.9 \\
\hline$[25]$ & $1.02 \pm 0.09$ & 2 & 24.6 & $18.6 \pm 4.5$ & spoon-left/surface breakup & 18.0 & 13.6 \\
\hline
\end{tabular}

TABLE 3. Selected disturbance data for ICE Mir vessel 2. $\omega_{n}$ is determined using a half-period. Characteristic lower cylinder radius $R=25.4 \mathrm{~mm}$ is used to determine $B o_{\omega}$. ${ }^{*}$ Only two peaks are used to compute $A_{\max }$.

average amplitude $A_{\text {ave }}$ for these disturbances are approximately $13.9 \mathrm{~mm}$ and $10.1 \mathrm{~mm}$, respectively. The disturbances do not give rise to any global reorientation of the liquid; the surface returns to the spoon-right configuration each time.

[14-15] The brief oscillation 14 of nearly two full periods (at $1.0 \mathrm{~Hz}$ ) has an increased amplitude of $A_{\max } \approx 24.8 \mathrm{~mm}$ with $A_{\text {ave }} \approx 18.9 \mathrm{~mm}$. The disturbance 14 produces a potato-chip-like configuration that is unstable. It holds on for $7 \mathrm{~s}$ during which it slowly retreats to the initial spoon-right configuration (15).

[16-18] Single period disturbances of amplitude $18.6 \mathrm{~mm}$ again result in a spoon-right configuration.

[19-22] Four complete periods of oscillations 19 at $0.84 \mathrm{~Hz}$ with $A_{\max } \approx 23.1 \mathrm{~mm}$ and $A_{\text {ave }} \approx 20.8 \mathrm{~mm}$ produce a potato-chip configuration that is stable (20). The configuration is shown in figure $9(c)$ with an orthogonal view shown in figure $9(d)$. The saddle-shaped interface resembles the potato chip predicted computationally (figures $3 b$ and $3 d$ ). It is given $60 \mathrm{~s}$ to stabilize before pulse-type disturbances $21-22$ of various amplitudes up to $17.8 \mathrm{~mm}$ are imparted over an additional $35 \mathrm{~s}$. Return of the surface to the chip after disturbances 21-22 indicates that a locally stable interface has been found. Small, spurious disturbances to the chip configuration result in surface waves of $\omega_{n} \approx 1.18 \mathrm{~Hz}, A<0.3 \mathrm{~mm}$, and $t_{s} \approx 4.2 \mathrm{~s}$.

[23-24] A more-than-three-period oscillation at $0.93 \mathrm{~Hz}$ with $A_{\max } \approx 25.6 \mathrm{~mm}$ and $A_{\text {ave }} \approx 19.4 \mathrm{~mm}$ destabilizes the chip. The surface shifts to a spoon, but now on the left side of the vessel as shown in figure $9(e)$. This shift requires $7 \mathrm{~s}$ after the destabilizing disturbance 23 is terminated and approximately $3 \mathrm{~s}$ more to stabilize (24).

[25-26] The large oscillation 25 imparted to the spoon-left surface at $1.0 \mathrm{~Hz}$ results in break-up of the surface. This disturbance is comparable in amplitude with the oscillations 19 that formed the stable chip, but is of higher frequency. Further disturbances of such magnitude lead to complete wetting of the interior surface of the vessel, after which the fluid breaks up into three elements: in the lid, in the bulge region, and at the base of the container, as shown in figure $9(f)$.

Table 3 summarizes some quantitative characteristics of the surfaces. Measurements from the disturbance history of figures 7 and 8 could be made with good accuracy for instantaneous frequency (to within 3\%) and amplitude (to within 2\%). However, the standard deviations of the respective average values are respectively $10 \%$ and $23 \%$ of the values, indicating that it was easier for the crew member to maintain a natural frequency than to maintain a constant amplitude. The link between increasing disturbance 


$\begin{array}{lccc}\text { Interface } & A_{\max } & \omega_{n} & t_{s} \\ \text { Configuration } & \mathrm{mm} & \mathrm{Hz} & \mathrm{s} \\ \text { initial symmetric } & 0.8 & 0.90 & 8.0 \\ \text { spoon-right } & 0.3 & 1.57 & 3.0 \\ \text { potato chip } & 0.3 & 1.18 & 4.2\end{array}$

TABle 4. Surface responses to small spurious disturbances, ICE Mir vessel 2.

amplitude and decreasing natural frequency for the large amplitude oscillations also contributed to the difficulty. Nonetheless, the uncertainties for the averaged values may be deemed respectable, as the disturbances are imparted by hand.

In the space experiments there were no attempts to induce the computed locally stable lichen configuration (figure $3 c$ ) to form. We considered inclusion of such attempts to have been beyond the scope of the resources available for the investigation.

\section{Discussion}

The process by which liquid reoriented from one configuration to another varied with the types of configurations. For the initial symmetric configuration (figure 9a), small spurious disturbances to the vessel resulted in global reorientation to the spoon configuration (figure $9 b$ ), while large amplitude axial oscillations were necessary to transform the spoon into the potato-chip configuration (figure $9 c$ ). The reorientation to the spoon from either the symmetric or potato-chip configuration was initiated by disturbances that largely died out before the bulk reorientation commenced. In contrast, the formation of the stable potato-chip configuration from the spoon (19 in figure 7) required first that a dynamic interface be produced with spatial periodicity close to that of the potato chip. Large amplitude axial oscillations were necessary to establish this interface. Once the oscillatory disturbances to the vessel ceased, the interface was apparently sufficiently near to the appropriate local energy well that it then settled easily into the chip configuration.

The time required for the bulk of the reorientation from the initial symmetric configuration to the spoon was about $3 \mathrm{~s}$, with another $3 \mathrm{~s}$ required for the last small part of the adjustment; the time for the transition from the potato chip to the spoon was longerabout $7 \mathrm{~s}$, plus another $3 \mathrm{~s}$ for final adjustment. One could interpret the shorter initial reorientation time as indication of a steeper energy well. Additionally, because the exotic container shapes near the bottom of the bulge vary so little with contact angle (figure $1 a$ ), the initial symmetric configuration is very close to being, if not actually, unstable. In any event, one can conclude for the $70^{\circ}$ liquid that the initial symmetric configuration is of substantially larger energy than the spoon configuration and that the potato chip's energy is intermediate between the two. These interpretations are in accord with the relative energies of the computed solution surfaces for such configurations (figure 3).

The magnitude of the disturbances necessary for transition between configurations to take place may be characterized by the dynamic Bond number $B o_{\omega}=\rho A \omega_{n}^{2} R^{2} / \sigma\left(\omega_{n}\right.$ in $\operatorname{rad~s}^{-1}$ ). This quantity is a measure of the ratio of excitation to restoration forces and is tabulated for the different disturbances in Table 3. For the values of $B o_{\omega}$ listed in the table, the radius of the lower cylinder $R=25.4 \mathrm{~mm}$ is used as characteristic length. From $A, \omega_{n}$, and $B o_{\omega}$ one obtains a measure of the strength of the input disturbance required for transition from one configuration to another. From Table 3 it is possible also to note the change in natural frequency with amplitude (see events 3,5 , and 7 ), an indication of the nonlinear character of oscillations of the interface. It should be emphasized that 
several of the amplitudes of the surface oscillations are quite large, with $A / R \approx 1$. The breakup of the surface occurred for a disturbance with average dynamic Bond number of approximately 14 .

In Table 4, the dynamic interface responses to small amplitude disturbances are given for the three configurations discussed above. These spurious disturbances were similar in nature and resulted from certain handling operations by the astronaut during the experiment. The input amplitudes of such disturbances were approximately $0.3 \mathrm{~mm}$ and can be treated as giving rise to small amplitude (linear) oscillations, $A / R \approx 0.01$. The natural frequencies and characteristic settling times were different for the different configurations. It was found for most cases that the smaller the natural frequency of the surface, the longer the settling time. The extremes can be noted by comparison of the entries in the table for the initial symmetric configuration and the spoon-right configuration; the frequency for the former is only about $57 \%$ of that for the latter, and the settling time is about 2.7 times as large. Additionally, one sees that the small disturbances produced significantly larger amplitude free surface oscillations for the initial symmetric configuration than for the asymmetric configurations. By comparison, the fundamental natural frequency in zero gravity for a right circular cylinder, without bulge, for a liquid with planar interface $\left(90^{\circ}\right.$ contact angle) approaches $2.3 \mathrm{~Hz}$ as the liquid depth becomes infinite (see Reynolds \& Satterlee 1966, p. 415).

It is important to note that the above discussion concerning behavior of transitions between the various configurations is for situations in which the requirements for an exotic container are generally not satisfied. In this connection we depict in figure 10 the locallystable spoon and potato chip configurations in a $55^{\circ}$ zero-gravity exotic container for both a liquid with $55^{\circ}$ contact angle and one with $70^{\circ}$ contact angle, as computed numerically with the Surface Evolver software. Each case is for a volume of liquid corresponding to the respective planar fill level (as in the procedures specified in $\S 4$ ). Generally, the surfaces observed in the experiments are consistent with the calculations in that they bear closer resemblance to the $70^{\circ}$ liquid case than to the $55^{\circ}$ one. What is essential, however, is not a precise such comparison but simply that the $70^{\circ}$ liquid produced asymmetric locallystable configurations in a container that is rotationally symmetric but is not necessarily an exotic one for that liquid.

Even if the conditions for an exotic container were closely satisfied, once the fluid becomes asymmetric the presence of symmetric equilibria nearby the initial one probably has little bearing on the fluid's subsequent behavior. In the experiments, the presence of the continuum of distinct symmetric equilibria likely has its main effect just in rendering the initial symmetric configuration less stable. It is of interest to note that in the mathematical proof of the instability of the planar member of the exotic continuum family it is the local boundary curvature that enters; if this curvature is greater than a certain lower bound, then the interface can be shown to be mathematically unstable, whether or not the container is exotic. (See Concus \& Finn 1989 and Finn 1988; a proof of instability that extends to all gravity levels appears in Wente 1999.)

\section{Concluding remarks}

What began for us as a study of nonuniqueness and of exotic containers has led to our encountering the striking phenomenon of the asymmetry of energy minimizing liquid configurations in containers that are rotationally symmetric and for which the prescribed boundary data (contact angles) are also symmetric.

Summarizing the experimental results from $\S 5$ and $\S 6$, we recount that two distinct locally stable nonaxisymmetric interfaces were observed in an axisymmetric container. 

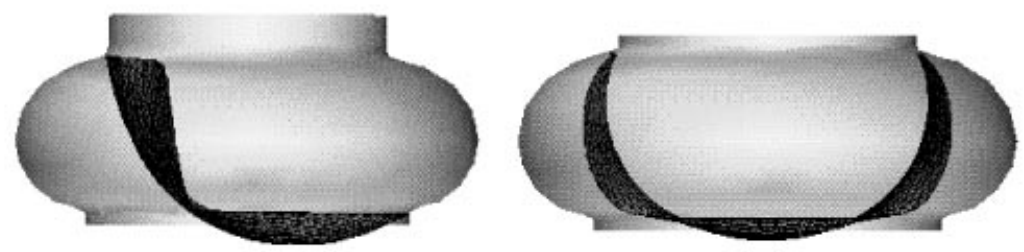

(a)
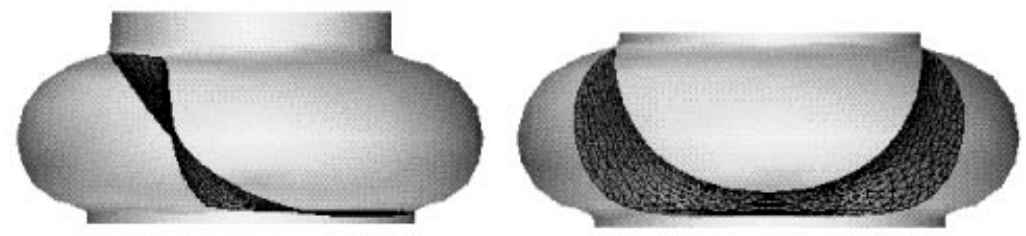

(b)

Figure 10. Computed spoon and potato chip configurations in a $55^{\circ}$ zero-gravity exotic container: (a) $55^{\circ}$ liquid, (b) $70^{\circ}$ liquid.

Four equilibrium surfaces in all were formed for ICE Mir prior to breakup, as depicted in figure 9. First the rotationally symmetric configuration observed during the initial filling, then the apparent global energy minimizer that was also found computationally (spoon), then (following further carefully applied disturbances) another local minimizer that was also found computationally (potato chip). Finally, a further disturbance led once more to the spoon, this time in reflected configuration. These surfaces are in general accord with the computed ones in figures 3 and 10 .

It was found additionally that only a small perturbation to the initial symmetric surface was necessary to destabilize it into an asymmetric configuration, whereas larger amplitude oscillations were necessary to reorient the liquid between the asymmetric configurations. Resonant frequencies and viscous settling times for the various configurations were found to differ by factors of approximately 1.7 and 2.7 , respectively.

There is suggestive evidence from other experiments (e.g. Concus, Finn \& Weislogel 1999; Langbein, Großbach \& Heide 1990) that perturbations may not be necessary for achieving energy minimizing configurations corresponding to the idealized equilibrium contact angle of the YLG theory; given sufficient time (which in particular cases may amount to hours or sometimes days), the fluid reorients itself, apparently under forces associated with Kelvin equilibrium, the thermodynamic balance between evaporation and condensation.

The accumulated evidence of the present and of other experiments suggests strongly that in cases of physical interest the YLG theory reliably predicts fluid behavior in lowgravity environments, or otherwise when surface forces predominate. Although in some cases the application of perturbations (or simply waiting long enough, as noted above) may be needed to overcome the effects of contact angle hysteresis, the fluid configurations finally adopted do appear to conform to predictions of the theory. The evidence suggests additionally that only in particular, perhaps rare, circumstances will capillary surfaces be 
uniquely determined by the data. Nor can it be expected that symmetric data will lead to symmetric configurations. In fact, as discussed above, even though the specifications for the exotic containers cannot be met exactly in practice, locally stable asymmetric surfaces will appear in them. Such surfaces may occur even for configurations that are distant from being exotic. These results can be important to designers of fluid management systems in low-gravity. The experimental results lend support to use of the classical YLG theory in such design procedures, and it must be noted that striking or unexpected behavior predicted by that theory will actually be observed in practice.

We are indebted to astronauts Lawrence DeLucas (USML-1) and Shannon Lucid (Mir) for the expert manner in which they conducted the space experiments; we wish to thank them for the detailed effort and thought they devoted to this investigation. We wish to thank also the U. S. National Aeronautics and Space Administration and the Russian Space Agency for the opportunity to use, respectively, the Space Shuttle and the Mir Space Station. Additionally, we wish to thank Victor Brady for carefully carrying out numerous computational experiments, which gave first glimpses of the phenomena under study, and for preparing the graphical output shown in figure 10. This work was supported in part by the National Aeronautics and Space Administration under Grants NCC3-329 and NAG3-1941, by the National Science Foundation under Grants DMS-9401167 and DMS-97029817, and by the Mathematical Sciences Subprogram of the Office of Energy Research, U. S. Department of Energy, under Contract Number DE-AC03-76SF00098.

\section{REFERENCES}

Brakke, K. 1996 Surface Evolver. Susquehanna Univ., Selinsgrove, PA, USA; available from http://www.geom.umn.edu/software/evolver.

Callahan, M., Concus, P. \& Finn, R. 1991 Energy Minimizing Capillary Surfaces for Exotic Containers. In Computing Optimal Geometries (with accompanying video tape) (ed. J. E. Taylor), AMS Selected Lectures in Mathematics, Amer. Math. Soc., pp. 13-15.

Concus, P. \& Finn, R. 1974 On Capillary Free Surfaces in a Gravitational Field. Acta Math. 132, 207-223.

Concus, P. \& Finn, R. 1989 Instability of Certain Capillary Surfaces. Manuscr. Math. 63, 209-213.

Concus, P. \& Finn, R. 1990 Capillary Surfaces in Microgravity. In Low-Gravity Fluid Dynamics and Transport Phenomena (ed. J. N. Koster \& R. L. Sani), Progress in Astronautics and Aeronautics, vol. 130, AIAA, pp. 183-205.

Concus, P. \& Finn, R. 1991 Exotic Containers for Capillary Surfaces. J. Fluid Mech., 224, 383-394; Corrigendum. J. Fluid Mech. 232, 689-690.

Concus, P., Finn, R. \& Weislogel, M. 1992 Drop-Tower Experiments for Capillary Surfaces in an Exotic Container. AIAA J. 30, 134-137.

Concus, P., Finn, R. \& Weislogel, M. 1993 Interface Configuration Experiment: Preliminary Results. In Joint Launch + One Year Science Review for USML-1 and USMP-1 with Microgravity Measurement Group, NASA CP-3272, vol. 2, pp. 525-541.

Concus, P., Finn, R. \& Weislogel, M. 1999 Measurement of Critical Contact Angle in a Microgravity Space Experiment. Exps. Fluids (in press).

FinN, R. 1986 Equilibrium Capillary Surfaces. Springer.

Finn, R. 1988 Nonuniqueness and Uniqueness of Capillary Surfaces. Manuscr. Math. 61, 347372.

Gulliver, R. \& Hildebrandt, S. 1986 Boundary Configurations Spanning Continua of Minimal Surfaces. Manuscr. Math. 54, 323-347.

Langbein, D., Grossbach, R. \& Heide, W. 1990 Parabolic Flight Experiments on Fluid Surfaces and Wetting. Microgravity Sci. Technol. 2, 198-211. 
Reynolds, W. C. \& Satterlee, H. M. 1966 Liquid Propellant Behavior at Low and Zero g. In The Dynamic Behavior of Liquids in Moving Containers (ed. H. N. Abramson), NASA SP-106, pp. 387-439.

Wente. H. 1999 Stability Analysis for Exotic Containers. Dynam. Contin. Discrete Impuls. Systems 5, 151-158. 